\title{
An Universal Approach for Choosing the Optimal Model when Studying any Physical Phenomenon
}

\section{Menin B* \\ Mechanical \& Refrigeration Consultation Expert, Israel}

*Corresponding author: Boris Menin, Mechanical \& Refrigeration Consultation Expert, Beer-Sheba 8464209, Israel, Tel: +972502205234; Email: meninbm@gmail.com

\section{Conceptual Paper}

Volume 3 Issue 4

Received Date: October 09, 2019

Published Date: October 22, 2019

\section{Abstract}

The problem of choosing the optimal model of a physical phenomenon or technological process is considered from the point of view of an information-oriented approach. This article proposes a new algorithm with which the minimum achievable uncertainty of the model and the required number of variables taken into account are calculated. Based on the analysis of scientific and technical publications, it is concluded that existing optimization methods do not provide a universal approach to this problem. We proceeded from the hypothesis that any model contains a certain amount of information about the studied object. The results confirm the original hypothesis.

Keywords: Technological process; Physical phenomenon; Information-oriented approach; Optimization methods

- What do you think about Newton's third law?

- I did not watch, I did not like the first two.

\section{Introduction}

When you ask whether you believe it or not, you come to the conclusion that over the past 20 years, huge share of scientific and technical articles cannot be refuted or confirmed. Original ideas, designs of various equipment, various technological processes developed by talented scientists and engineers are presented by models in scientific works without comparing the magnitude of the experimental uncertainty achieved with the difference between the theoretical curves data and the results of experiments. To paraphrase the famous quotation of Lenin, how far all these models are from the mass of people. It would seem that this requirement is the basis of the advancement of theoretical thought into practice. The percentage of talented articles is decreasing. So what has a greater impact on scientific progress: theory, experiment or the desire to increase your citation?

The author, being an engineer with 46 years of experience and a specialist in heat and mass transfer in refrigeration units, as well as specializing in the modeling of physical phenomena and technological processes, undertook an ungrateful job: to analyze the results of scientific and applied articles in recent years, at least in areas in which he understands to some extent.

As was proved in Menin B [1], a necessary condition for the eligibility of the model is the small value of the calculated total uncertainty of the measurement of the objective function compared to the difference between theoretical data and experimental results. Therefore, two analyzes of scientific and technical articles were carried out. 


\section{Physical Science \& Biophysics Journal}

In Menin B [2] analysis of theoretical and experimental studies of cold energy storage systems published between 2000 and 2016 was organized. Selection of data was based on the following criteria:

1. The presence of a comparison of experimental results with theoretical calculations or computer simulations.

2. The representation of numerical values of the absolute or relative uncertainties of calculation or measuring the value of the main researched variable.

3. The presence of charts or tables with declared measuring ranges or computer simulations.

Keywords of the search were "measurement", "uncertainty", "mathematical model", "experiments" and "cold energy storage system". The chosen interval of publications was $2000-2016$.

According to these criteria, only twelve publications were selected. All developers claimed that the achieved results confirm the theoretical models presented. However, in no work there were specific numerical values of the achieved overall measurement uncertainty of the similarity criterion for the main researched function or summary criteria. In fact, none of the studies determines and calculates the generalized absolute uncertainty of the main variable. No one compares the difference between numerical forecasts and experimental results with the calculated absolute uncertainty of the main variable.

The following search was devoted to studying the content of articles presented in the International Journal of Refrigeration from 2014 to 2019. A total of 1569 articles were analyzed. For free reading of articles the SCIHUB tool was used. Articles were screened based on three criteria: a. the presence of a comparison of experimental results with a database obtained in theoretical and / or computer modeling; $b$. the presence of the calculated achieved value of the measurement absolute or relative uncertainties; c. the availability of information on the intervals of variation of the main studied variable and other variables taken into account in the developed model. By these criteria, only 317 (20.2\%) publications were found.

By these criteria, only 317 (20.2\%) publications were found. Moreover, in all filtered articles, the authors announced that the discrepancy between theoretical predictions and experimental data is insignificant. When analyzing these articles, the impression was made that scientists, exploring physical phenomena and technological processes and using modern mathematical apparatus and powerful computers in their daily work, perceived their ideas as reality and affirmed the validity of their models, not understanding with what actual accuracy they declared their results.

Presenting the chapter "Uncertainty Analysis" or "Instrumental Uncertainties", the authors, in essence, reported only information on the accuracy of measuring devices or calculated the measurement uncertainty by dividing theoretical data by experimental results. A detailed example of calculating the total uncertainty of the main variables was presented in only one article. In scientific practice, it is accepted that the researcher confirms the formulated idea by comparing theory with experiment. In fact, in most publications there is no information about the calculated values of the general uncertainty of measurement of the studied variable or dimensionless complex.

It is difficult to believe that the calculation of various types of uncertainties and their influence on the accuracy of the model is stated in only one document [3]. The authors of publications did not bother to compare the experimental uncertainty of measurement of the studied variable (EU) (its definition was introduced in Tian $\mathrm{Z}, \mathrm{Gu}$ B, Qian C, Yang L, Liu F [4]) with the difference between theoretical (TD) and experimental data (ED). If the EU> | TD - ED |, then the model is erroneous, and not applicable in practice. The only gain from publishing such an article is a researcher who increases his own citation index, and the editorial board of the journal that receives publication fees. An analysis of these publications leads us to a disappointing conclusion. Neglect of the methods of validation and verification and analysis of uncertainties will lead to a catastrophic situation: the number of researchers and articles published by them is steadily growing, and the practical significance of theoretical studies is falling. Thus, without a doubt, a prerequisite for the validity of the model is to compare the discrepancy between the theoretical and experimental results with the calculated, achieved experimental uncertainty in the measurement of the desired studied variable: $\mathrm{EU} \sim$ |TD $\mathrm{ED}$. As we show below, this experimental absolute uncertainty, in turn, must be compared with the theoretically achievable one, calculated in accordance with the information-oriented method.

These two analyzes are related to refrigeration. Although one can suspect that the same depressing, one might even say catastrophic, situation is characteristic of other areas of technology and science. Question by Nikolai Chernyshevsky, Russian writer: what to do? - is relevant today. 


\section{Physical Science \& Biophysics Journal}

In modeling physical processes, the uncertainty principle [5] has profound implications for our view of the world, although it is still the subject of many contradictions. The uncertainty principle means that it is impossible to accurately reflect the observed process in mathematical formulas. Of course, you can use powerful computers and complex algorithms with a huge number of variables. However, each variable has its own uncertainty, causing non-zero (maybe even greater) model uncertainty. Therefore, it can be assumed that there is an optimal number of variables to achieve minimal model uncertainty. At the same time, all existing analytical and statistical methods, algorithms and techniques that use various optimization criteria are focused on achieving minimal uncertainty of the model after its formulation and in the process of computerization. In this case, the initial uncertainty due to the number of variables in the model and their qualitative set is completely ignored, that is, what process or phenomenon these variables are intended to reflect: heat and mass transfer, mechanics, electromagnetism, and so on. This gap is filled by the recently proposed information-oriented method, the various applications of which this study is devoted.

\section{Initial Thoughts}

Various criteria are used to determine the distance between theoretical results and experimental data. This discrepancy is valid for many pairs of distance metrics and is a problem for engineers and scientists who are trying to develop the most efficient algorithms. This means that an algorithm that works for one type of distance will not work for another, that is, until a new search method appears. According to the author, the information approach is a theoretically justified universal method for comparing theoretical results and experimental data. This is explained by the following.

In this case, the author moved away from the pursuit of specific algorithms and criteria for the smallest gap. Instead, he asked broader questions: what influences the choice of the threshold discrepancy [6] algorithm for calculating the distance metric? b. What prevents a good algorithm from being universal for a wide range of studies? The answers, according to the author, are related to the amount of information contained in the model and to the situation in which (the scientific community neglects it) it is possible and necessary to find the best model in accordance with the "class of phenomenon" (CoP). CoP indicates a situation where the selected variables are associated with a particular physical phenomenon or process. For example, when simulating an airplane's flight, it is necessary to take into account the variables of the International System of Units (SI) with dimensions of mass, $\boldsymbol{M}(\mathrm{kg})$, length, $\boldsymbol{L}(\mathrm{m})$, time, $\boldsymbol{T}(\mathrm{s})$, temperature, $\boldsymbol{\theta}\left({ }^{0} \mathrm{~K}\right)$ and air, $\boldsymbol{F}(\mathrm{mol})$, that is why $\mathrm{CoP} \equiv$ LMTEF. The new algorithm is important not only theoretically, but also in practice, since it is much easier to learn and simpler than the algorithms that are already used.

The central problem of modeling is to decide how many variables to apply to the model and their qualitative set. Numerous studies have shown that maintaining too few or too many variables can be detrimental to the interpretation of the results predicted by the model, so choosing the optimal number of variables for a particular CoP is extremely important.

The objectives of this study are (a) to propose using the model selection approach with regard to determining the number of variables, (b) to offer a theoretical basis that will help guide the decision-making process, and (c) to present a universal criterion for choosing the optimal number of variables in the model.

Although the true model does not exist, the search for the optimal finite number of variables in the model is possible using the information method. The optimal number of variables is understood to be such a value at which the minimum absolute uncertainty of the desired variable under study can be achieved with the selected CoP. However, we must remember that any method that cannot be implemented or understood by the scientific community will not receive approval. This implies that, at least, we need a method that can be easily performed and that produces results that can be interpreted by scientific and competent end users. In addition, from the point of view of a scientist or engineer, the method should be sufficiently general to solve a wide range of physical and technical problems. And from the point of view of information theory, it is important that the method be connected with the principle of probability and have some kind of frequency justification.

From the above positions, let us look at SI. In all areas of science and technology, scientists use SI to implement their ideas. SI is inherently a new element of scientific knowledge, completely alien to classical concepts. It exists only thanks to the consensus of researchers, although SI is absent in nature. SI includes the base and derived quantities used to describe the various classes of phenomena (CoP). In other words, the limits of the 


\section{Physical Science \& Biophysics Journal}

description of the studied material object are determined by the choice of $\mathrm{CoP}$ and the number of derived quantities taken into account in the mathematical model [7]. In addition to the above five base variables (quantities) ( $\boldsymbol{L}$, $\boldsymbol{M}, \boldsymbol{T}, \boldsymbol{\theta}, \boldsymbol{F})$, consideration of the processes of electromagnetism adds the magnitude of the electric current $\boldsymbol{I}$. For photometry, it is necessary to add the J-light intensity [8].

To represent the dimension of any derived variable $\boldsymbol{q}$, a unique combination of dimensions of the main base variables with various degrees is used [10]:

$$
\boldsymbol{q} \ni \boldsymbol{L}^{l} \cdot \boldsymbol{M}^{m} \cdot \boldsymbol{T}^{t} \cdot \boldsymbol{I}^{i} \cdot \boldsymbol{\Theta}^{\Theta} \cdot \boldsymbol{J}^{j} \cdot \boldsymbol{F}^{f}
$$

where

1. $l, m, \ldots f$ are exponents of the base quantities and the range of each has a maximum and minimum value; according to [9], integers are the following:

$$
\begin{gathered}
-3 \leq l \leq+3,-1 \leq m \leq+1,-4 \leq t \leq+4,-2 \leq i \leq+2 \\
-4 \leq \theta \leq+4,-1 \leq j \leq+1,-1 \leq \mathrm{f} \leq+1
\end{gathered}
$$

2. Because the exponents of the base quantities can only take integer values, the number of choices of dimensions for each quantity $\boldsymbol{e}_{l}, \ldots \boldsymbol{e}_{f}$, according to (2), is the following:

$$
e_{l}=7 ; e_{m}=3 ; e_{t}=9 ; e_{i}=5 ; e_{\theta}=9 ; e_{j}=3 ; e_{f}=3 \text {. }
$$

where, for example, $\boldsymbol{L}^{-3}$ is used in a formula for density, and $\Theta^{4}$ in the Stefan-Boltzmann law;

3. The total number of dimension options of physical quantities equals $\boldsymbol{\Psi}^{\circ}=\prod_{l}^{f} \boldsymbol{e}_{i}-1$

$$
\boldsymbol{\Psi}^{\circ}=\left(\boldsymbol{e}_{l} \cdot \boldsymbol{e}_{m} \cdot \boldsymbol{e}_{t} \cdot \boldsymbol{e}_{i} \cdot \boldsymbol{e}_{\theta} \cdot \boldsymbol{e}_{j} \cdot \boldsymbol{e}_{f}-1\right)=76,544, \text { (4) }
$$

where " -1 " corresponds to the case where all exponents of the base quantities in formula (1) are treated as zero dimension, $\prod$ is a product of elements $\boldsymbol{e}_{l}, \ldots \boldsymbol{e}_{f}$;

4. The value $\Psi^{\circ}$ includes both required and inverse quantities (for example, $L^{1}$ is the length, $L^{-1}$ is the running length). The object can be judged knowing only one of its symmetrical parts, while others structurally duplicating this part may be regarded as information empty. Therefore, the number of options for dimensions may be halved. This means that the total number of dimension options of physical quantities without inverse quantities equals $\Psi=\Psi^{\circ} / 2=38,272$.

According to the $\pi$-theorem [10], the number $\boldsymbol{\mu}_{\mathrm{SI}}$ of possible dimensionless criteria with $\xi=7$ base quantities for SI will be:

$$
\boldsymbol{\mu}_{\mathrm{SI}}=\boldsymbol{\Psi}-\xi=38,265
$$

Equation (5) was obtained for the first time [1], and its existence can be considered as a statement that SI is a kind of multidimensional space that includes all possible dimensions of variables used in science. Our starting point is that the measuring space of the object under study has one emerging holographic direction in the form of equation (1), including seven base variables. Additional assumptions are that (i) the entropy changes in the arising direction when formulating the object model, (ii) the number of degrees of freedom depends on the chosen class of phenomena, and (iii) information about the object is evenly distributed among all these variables. That is, the researcher's choice of a variable in the model, on the one hand, is equally probable, and on the other hand, depends on the willful decision of the scientist, who is guided by his experience, knowledge and intuition. Thus, one and the same phenomenon can be analyzed by various research teams using groups of variables that are different in quantity and quality set. The most famous example of such a situation is the consideration of an electron as particle or wave.

The uniqueness of the result is that equation (5) is valid for any physical phenomenon or technological process. This is not even a hypothesis, but a rule that, along with the choice of a specific class of phenomena, must be taken into account when formulating the model. Of course, one cannot be sure that in the future (near or far) an additional base quantity will be introduced.

Then, taking into account Equation (5) and the earlier statement about the equally probable choice of quantities when formulating the model, we can calculate the amount of entropy in SI $[1,11]$ :

$$
\mathbf{H}=\boldsymbol{k}_{\mathrm{b}} \cdot \ln \boldsymbol{\mu}_{\mathrm{SI}},(6)
$$

where $\mathbf{H}$ is the entropy of SI including $\boldsymbol{\mu}_{\mathrm{SI}}$, equally probable accounted quantities, and $\boldsymbol{k}_{\mathrm{b}}$ is the Boltzmann constant. 


\section{Physical Science \& Biophysics Journal}

Given equation (5), it was proved [1] a new rule, called $\boldsymbol{\mu}_{\mathrm{SI}}$-rule in formulating a model of any physical phenomenon or process can be defined as follows:

when formulating the measurement model of the dimensionless variable $\mathbf{u}$, which takes values in a given range $S$, we always use a system of basic quantities with a total number of dimensional physical variables, $\Psi$, where $\xi$ of which have independent dimensions, which means $\boldsymbol{\mu}_{\mathrm{SI}}=\Psi-\xi$. When choosing a class of phenomena ( $\mathbf{z}^{\prime}$ is the total number of dimensional variables, and $\boldsymbol{\beta}^{\prime}$ is the number of basic variables) and a measurement model (a given number of physical dimensional variables $\mathbf{z}$ " taken into account in the model, where $\boldsymbol{\beta}^{\prime \prime}$ of which is the number of selected base variables in the model), the absolute measurement uncertainty of the main variable $\Delta_{\mathrm{pmm}}$, can be determined from the relation:

$$
\boldsymbol{\Delta}_{\mathrm{pmm}}=S \cdot\left[\left(\boldsymbol{z}^{\prime}-\boldsymbol{\beta}^{\prime}\right) / \boldsymbol{\mu}_{\mathrm{SI}}-\left(\boldsymbol{z}^{\prime \prime}-\boldsymbol{\beta}^{\prime}\right) /\left(\boldsymbol{z}^{\prime}-\boldsymbol{\beta}^{\prime}\right)\right],
$$

where $\varepsilon=\boldsymbol{\Delta}_{\mathrm{pmm}} / \boldsymbol{S}$ is the comparative uncertainty [11].

It inevitably follows from this that the resulting smallest model uncertainty (blurriness of the image of a real object) is determined by equation (7). Additionally, the reader must remember, to determine the minimal uncertainty, we do not need the details of the information about a structure of the model, only the amount of information provided by the change in entropy with a decrease in the number of variables taken into account compared to the total number of variables in the SI and a declaration of the chosen CoP.

Equation (7) is very simple, although it is not a purely mathematical abstraction and has physical meaning: in nature there is a fundamental limit to the accuracy of measurement of any process, which cannot be surpassed by any improvement of tools, measurement methods or computerization of the model. The value of this limit is much stronger than the Heisenberg uncertainty relation gives. Equation (7) can be used to analyze any experimental results with both dimensional and dimensionless variables due to the following relations

$$
\begin{gathered}
\Delta U / S^{*}=(\Delta U / a) /(S * \backslash / a)=(\Delta u / S)(8) \\
(\boldsymbol{r} / \boldsymbol{R})=(\boldsymbol{\Delta U} / \boldsymbol{U}) /(\boldsymbol{\Delta u} / \boldsymbol{u})=(\boldsymbol{\Delta U} / \boldsymbol{U}) \cdot(\boldsymbol{a} / \boldsymbol{\Delta U}) \cdot(\boldsymbol{U} / \boldsymbol{a})=
\end{gathered}
$$

1 ,

where $\boldsymbol{S}$ and $\boldsymbol{\Delta u}$ are the dimensionless quantities (respectively, the range of variations and the total absolute uncertainty in determining the dimensionless quantity $\boldsymbol{u}) ; \boldsymbol{S}^{*}$ and $\boldsymbol{\Delta U}$ are the dimensional quantities (respectively, the range of variations and the total absolute uncertainty in determining the dimensional quantity $\boldsymbol{U}$ ); $\boldsymbol{a}$ is the dimensional scale parameter with the same dimension as that of $\boldsymbol{U}$ and $\boldsymbol{S}^{*} ; \boldsymbol{r}$ is the relative uncertainty of the dimensional quantity $\boldsymbol{U}$; and $\boldsymbol{R}$ is the relative uncertainty of the dimensionless quantity $\boldsymbol{u}$.

\section{Examples}

\section{Ice Maker}

The article Murgham H, Miszka D, Wynn K [12] is of great interest both from a scientific and technical point of view. Simulation of refrigeration equipment with detailed analysis of heat and mass processes (more than 80 variables were used, and CoP $\equiv \boldsymbol{L M T \boldsymbol { \theta }}$ ) in an ice maker with subsequent experimental tests on a commercially available unit is very important. The approach used can be proposed to study other types of refrigeration equipment. The authors outlined the specific test conditions for the ice maker, indicated the boundary and initial conditions, and also presented a comparison between the theoretical calculation and the experimental results for the key performance indicators of the ice maker: freeze and harvest cycle times, ice per day, energy consumption per 100 pounds and energy consumption in day. The small, in the opinion of the authors, absolute difference in percentages between experiment and modeling (the authors called it "model's accuracy within 5 \%") allowed them to draw far-reaching conclusions regarding the possible improvement of the ice maker performance.

Returning to the data of the Introduction chapter, it can be argued that only $20 \%$ of the publications of the International Journal of Refrigeration for 2012-2018 correspond to this level of research, which combines theory, modeling, testing a well-established industrial ice generator and comparing theoretical and experimental data. However, guided by the principles of the information method, we are forced to add a fly in the ointment in a barrel of honey: it is difficult to agree with some of the authors' claims.

First of all, the model accuracy claimed by the authors and expressed as the difference between the theoretical calculation and the result of the experiment, is not the model uncertainty and cannot be used to confirm the validity of the theoretical model. If we assume that only three declared experiments were carried out under different operating conditions, then it is incredibly (!!!) not enough to calculate the mismatch between 


\section{Physical Science \& Biophysics Journal}

computational predictions (CP) and experimental results (ER), |CP - ER| (for example, its definition can use the mean absolute deviation $[13,14])$.

In addition, if experimental uncertainty $\mathrm{EU}>|\mathrm{CP}-\mathrm{ER}|$, the value of the proposed model is insignificant, and it is very risky to put it into practice, but the developer wins because she or he increases their own citation index. This is one of the reasons why designers, having seemingly sufficiently reliable models, are forced to increase, for example, the design cooling capacity of a compressor or the volume of a storage tank by at least $20-40 \%$.

An additional comment is related to the magnitude of the inaccuracy model achieved. Even if we assume that all $(\mathrm{N}=80)$ variables are calculated and measured with very high accuracy, for example $1 \%$ (it is very difficult to believe), then the total relative uncertainty achieved in the key performance criteria of the ice maker $\mathrm{rE}$, for example energy consumption in day, will be equal to [6]:

$$
r_{E}=K \cdot\left(\sum^{N}{ }_{i=1} r_{i}^{2}\right)^{1 / 2}=1.2 \cdot\left(80 \cdot 0.01^{2}\right)^{1 / 2}=1.2 \cdot 0.089=0.107
$$

where $\mathrm{K}$ is a correction factor equal to 1.2 for the most important parameters of the devices and monitoring the characteristics of the finished product.

This value (0.107) covers much of the declared model's accuracy (0.05), which confirms the problematicity or correctness of the proposed physical and mathematical model.

Let us verify the value of the comparative uncertainty recommended according to the information method for a particular CoP. In order to find out, we need to equate the derivative

$\boldsymbol{\Delta}_{\mathrm{pmm}}$ / S (7) with respect to $\boldsymbol{z}^{\prime}-\boldsymbol{\beta}^{\prime}$ to zero:

$$
\left(z^{\prime}-\boldsymbol{\beta}^{\prime}\right)^{2} / \boldsymbol{\mu}_{\mathrm{SI}}=\left(z^{\prime \prime}-\boldsymbol{\beta}^{\prime \prime}\right)
$$

For CoP $\equiv \boldsymbol{L M T \boldsymbol { \theta }}$ inherent in the model [12], the lowest comparative uncertainty can be reached at the following conditions:

$$
\begin{gathered}
\left(z^{\prime}-\boldsymbol{\beta}^{\prime}\right)_{L M T \theta}=\left(\boldsymbol{e}_{l} \cdot \boldsymbol{e}_{m} \cdot \boldsymbol{e}_{t} \cdot \boldsymbol{e}_{\theta}-1\right) / 2-4=846, \\
\left(z^{\prime \prime}-\boldsymbol{\beta}^{\prime \prime}\right)_{L M T \theta}=\left(z^{\prime}-\boldsymbol{\beta}^{\prime}\right)^{2} / \boldsymbol{\mu}_{\mathrm{SI}}=846^{2} / 38,265 \approx 19,
\end{gathered}
$$

where " 1 " corresponds to the case where all the base quantities exponents are zero in formula (1); 4 corresponds to the four base quantities $\boldsymbol{L}, \boldsymbol{M}, \boldsymbol{T}$, and $\boldsymbol{\theta}$; and division by 2 indicates that there are direct and inverse quantities, e.g., $\mathrm{L}^{1}$ is the length and $\mathrm{L}^{-1}$ is the run length. The object can be judged based on the knowledge of only one of its symmetrical parts, while the other parts that structurally duplicate this one may be regarded as information empty. Therefore, the number of options of dimensions may be reduced by a factor of two.

Then, one can calculate the minimum achievable comparative uncertainty $\varepsilon_{L M T \theta}$ :

$$
\varepsilon_{\text {LMTO }}=\left(\Delta_{\mathrm{pmm}} / S\right)_{\text {LMTO }}=846 / 38,265+19 / 846=0.0442 .
$$

For interval $S_{\mathrm{E}}$ of "the energy consumption in day" recorded in experiments and equaled $3.5 \mathrm{kWh}\left(S_{\mathrm{Emax}}-\right.$ $S_{\text {Emin }}$ [12] and the supposed its relative uncertainty equaled $\mathrm{r}_{\mathrm{E}}=0.107$ (9), experimental absolute $\Delta_{\exp }$ and comparative eexp uncertainties equal

$$
\begin{gathered}
\Delta_{\exp }=\mathrm{r}_{\mathrm{E}} \cdot\left(\left(S_{\mathrm{Emax}}+S_{\mathrm{Emin})}\right) / 2\right)=0.107 \cdot((23.75+20.25) / 2)=2.35 \\
\operatorname{kWh}(14) \\
\varepsilon_{\exp }=\Delta_{\exp } / S_{\mathrm{E}}=2.35 / 3.5=0.6714 .
\end{gathered}
$$

The value $(0.6714)$ is significantly higher than the recommended value $(0.0442)$, which once again casts doubt on the credibility of the proposed model.

Thus, the stated well correlation between the experimental and calculated data does not guarantee that the choice of the model structure will be sufficiently complete with the number of quantities taken into account. A model with formulated relationships between the main characteristics of the process under study may be incorrect and should be improved. For further experimental work, it is recommended to use devices of a higher accuracy class, sufficient to confirm or refine a new physical-mathematical model developed using a larger number of experiments.

In this example there was introduced the detailed step-by-step analysis of the physical-mathematical model based on information-oriented approach. 


\section{Physical Science \& Biophysics Journal}

\section{Thermal Storage System}

Using an informational approach, we will try to compare two different studies describing the same energy storage system in terms of their information content.

In [15] the charging and discharging characteristics of a laboratory prototype of a latent heat storage device for cooling were experimentally and numerically studied. Two numerical models ( $\mathrm{CoP} \equiv \boldsymbol{L M T \boldsymbol { \theta }}, \boldsymbol{z}^{\prime \prime}=35$ variables) were developed to analyze the operational characteristics of the LHTES prototype: a purely conductive model and a conductive-convective model. Performance parameters such as melt fraction, charge / discharge time and energy storage / discharge rate are evaluated. The authors announced the validation process of the developed numerical models for the process of charging and discharging, as well as changes in the local temperature in the prototype during computer simulation by comparing with the corresponding temperature values obtained from experiments. According to the results of only a qualitative comparison, the authors preferred the use of a conductive-convective model that better describes the phenomena of heat transfer both for the charging process and for discharge.

No information was provided about the uncertainty of the measured values of temperature, thermal conductivity, specific heat, latent heat of fusion, heat transfer coefficient, stored energy and other variables. Of course, this situation dramatically reduces the impression of the credibility of the proposed model.

In [16] the dynamic thermal characteristics of a hightemperature system for the accumulation of latent thermal energy contained in spherical capsules were

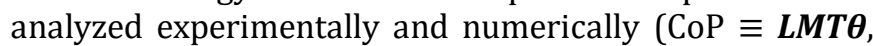
$\boldsymbol{z}^{\prime \prime}{ }_{2}=29$ variables). Spherical capsules are filled with sodium nitrate, and air is used as a coolant. Models with a transitional two-dimensional continuous solid phase and an effective compacted layer are developed and verified by comparison with experimental results. Using these models, the detailed heat transfer characteristics between the capsules and the coolant are analyzed.

The authors noted that the presented numerical models can be used to analyze detailed characteristics of the coolant flow in a system with a compacted layer and to obtain dimensionless correlations for a large-scale system.

Unfortunately, the authors mentioned only the measurement uncertainties of temperature and flow rate.
There were no quantitative calculations of the total uncertainty of the target variables, such as the total heat capacity and coolant flow rate. The declaration of a "good agreement between numerical and experimental" data is indicated only in graph form. Quantitative calculations in the difference between theory and field tests are not given. Therefore, the presented results are highly controversial. The authors did not take into account the fact that validation alone does not prove the proximity and consistency of theoretical data and experimental results [17]. Therefore, the results of visual validation cannot be a tool to prove the acceptability of the model.

For all this, in order to answer the question of which of these two studies is more informative into the frame of the information-oriented approach, the following should be taken into account. Any model of the investigated object contains a certain amount of information $\Delta \mathbf{A}_{\mathrm{e}}$ :

$$
\Delta \mathrm{A}_{\mathrm{e}}=\boldsymbol{\kappa} \cdot \ln \left[\boldsymbol{\mu}_{\mathrm{SI}} /\left(\boldsymbol{z}^{\prime \prime}-\boldsymbol{\beta} "\right)\right]
$$

where $\boldsymbol{\Delta} \mathbf{A}_{\mathrm{e}}$ is expressed in units of entropy [18], $\boldsymbol{z}^{\prime \prime}$ and $\boldsymbol{\beta}^{\prime \prime}$ are, respectively, the number of all and base quantities registered in a model, $\boldsymbol{k}$ is Boltzmann constant.

To calculate amount of information in bits $\Delta \mathbf{A}_{b}$ embedded in a model, it is necessary to divide $\Delta \mathbf{A}_{\mathrm{e}}$ by $\boldsymbol{\kappa} \ln 2=$ $9.569926 \cdot 10^{-24} \mathrm{~kg} \cdot \mathrm{m}^{2} \cdot \mathrm{s}^{-2} \cdot \mathrm{K}^{-1}[11,19]$ :

$$
\Delta \mathbf{A}_{\mathrm{b}}=\ln \left[\boldsymbol{\mu}_{\mathrm{SI}} /\left(\boldsymbol{z}^{\prime \prime}-\boldsymbol{\beta} "\right)\right] / \ln 2 \text {. (bits) (17) }
$$

Thus, to identify the most informative version when comparing two models describing the same energy storage system, the following formula should be used:

$$
\begin{aligned}
& \boldsymbol{\Delta} \mathbf{A}_{\mathrm{b} 12}=\ln \left[\boldsymbol{\mu}_{\mathrm{SI}} /\left(\boldsymbol{z}_{1} "-\boldsymbol{\beta}_{1} "\right)\right] / \ln 2-\ln \left[\boldsymbol{\mu}_{\mathrm{SI}} /\left(\boldsymbol{z}_{2} "-\boldsymbol{\beta}_{2} "\right)\right] / \ln 2= \\
& =\ln \left[\left(z_{2} "-\boldsymbol{\beta}_{2} "\right) /\left(\boldsymbol{z}_{1} "-\boldsymbol{\beta}_{1} "\right)\right] / \ln 2
\end{aligned}
$$

where $\mathbf{\Delta} \mathbf{A}_{\mathrm{b} 12}$ is an information amount difference between two models describing the same system, however, belonging to the same CoP; $\boldsymbol{z}_{1}$ " and $\boldsymbol{\beta}_{1}$ " are, respectively, the number of quantities and the number of the base quantities in the first model; $\mathbf{z}_{2}$ " and $\boldsymbol{\beta}_{2} "$ are, respectively, the number of quantities and the number of the base quantities recorded in the second model. If $\boldsymbol{\Delta} \mathbf{A}_{\mathrm{b} 12}<0$, then the second model contains more detailed information about the energy storage system under study. If $\boldsymbol{\Delta} \mathbf{A}_{b 12}>0$, then the first model is preferred. 


\section{Physical Science \& Biophysics Journal}

Using (18) and assuming that in each of the two models five base quantities are selected, i.e., $\boldsymbol{\beta}_{1}{ }^{\prime \prime}=\boldsymbol{\beta}_{2}{ }^{\prime \prime}$, we can calculate

$$
\boldsymbol{\Delta} \mathbf{A}_{\mathrm{b} 12}=\ln \left[\left(\boldsymbol{z}^{\prime}{ }_{2}-\boldsymbol{\beta}{ }_{2}\right) /\left(\boldsymbol{z}_{1}-\boldsymbol{\beta}{ }_{1}\right)\right] / \ln 2=[\ln (24 / 30)] / \ln 2=-0.32 \text {. }
$$

(bits) (19)

According to (19), the model [15] is less informative in comparison with [16]. This is explained by the fact that in the second model [16] the number of dimensionless criteria is closer to the optimal one - 19 , according to formula (12). Unfortunately, the lack of specific detailed data on measurement uncertainties does not allow us to give preference to any model. From the point of view of the information-oriented method, studies of the proposed models are presented in a form that does not allow us to unequivocally agree or reject the results achieved. Therefore, the author is convinced that the presented results would be more impressive if there were a detailed analysis of the calculated uncertainties of the studied variables in these studies.

Thus, the analysis of the introduced examples indicates a depressing and disappointing situation that has developed recently in scientific research publications. On the one hand, stunning achievements in mathematical methods and computer programs allow scientists and engineers to carry out very complex research in various fields of human activity. However, on the other hand, in practice, verification of the veracity and the possibility of reproducing their results, due to the lack of professional calculation of the accuracy of the experiment, leads to the devaluation of the study.

\section{Conclusion}

Since the researcher selects a model based on knowledge, experience, information obtained from other scientists, and intuition, regardless of how he does it, the model is only for guidance only and cannot confirm or refute the original idea. Confirming conclusions can only be made based on well-planned follow-up studies and verification of compliance or a significant difference between theoretical curves and experimental data. If theory and practice are close, valuable and reliable information can be obtained regarding the assumptions of the researcher and the predictions of the proposed model. Obviously, there are many practical considerations regarding the quantitative and qualitative choice of variables in the model, which to some extent determine the uniqueness of a particular model. Nevertheless, based on the stated principles of the information-oriented method, we can formulate general postulates:

A necessary condition for the satisfied accuracy of the model is, above all, the smallness of the calculated total uncertainty of the objective function (quantity) compared with the mismatch between the theoretical and experimental values. This fundamental truth has not had an important place in the scientific literature, but has must be the subject of considerable debate.

The fundamental limit of measurement accuracy of any variable that cannot be surpassed by any improvement in tools, measurement methods or computerization of the model is dictated by the $\boldsymbol{\mu}_{\mathrm{sI}}$-rule.

The value of the smallest comparative uncertainty in the measurement of the studied variable does not depend on the measurement process and is determined only by the researcher's choice of a particular class of phenomena.

The selected class of phenomena corresponds to a specific value of comparative uncertainty and the number of variables taken into account in the model. Therefore, $\boldsymbol{\mu}_{\mathrm{SI}}$-rule is a universal metric of choosing the optimal model of any physical phenomenon or technological process.

The proximity of theoretical predictions to experimental data is implied by the majority of researchers, as self-evident confirmation of the proposed theoretical concept. However, a "statistical significance" between the results of any type of theoretical model and experimental data is not sufficient evidence of the correctness of the chosen model [20].

Without a decisive change in the position of scientists and engineers regarding a more thorough and balanced calculation of uncertainties in measuring equipment parameters and process characteristics, the gap between a steady increase in the number of published scientific articles and their practical significance will increase.

Today it is easier to criticize - but it is much more necessary than in the past. Reliably verified data today play a much more important and much more central role. They are much more complex. Scientists need a tool with which they can look at themselves and test their ideas. This tool is an informational method. 


\section{Physical Science \& Biophysics Journal}

I hope that the readers-authors will find the opportunity to confirm their assumptions using the principles of the information-oriented method. On the other hand, my comments will be of interest to scientists and engineers with intellectual and computer potential. It is a long journey, but worth it.

\section{References}

1. Menin B (2017) Information Measure Approach for Calculating Model Uncertainty of Physical Phenomena. American Journal of Computational and Applied Mathematics 7(1): 11-24.

2. Menin B (2017) Preferred physical-mathematical model of the cold energy storage system. Applied Thermal Engineering 112: 1020-1026.

3. Kim HS et al. (2016) Flow characteristics of refrigerant and oil mixture in an oil separator. International Journal of Refrigeration 70: 206-218.

4. Tian Z, Gu B, Qian C, Yang L, Liu F (2015) Electronic expansion valve mass flow rate prediction based on dimensionless correlation and ANN model. International Journal of Refrigeration 57: 1-10.

5. Heisenberg, W (1927) About the descriptive content of quantum theoretical kinematics and mechanics. magazine for physics. 43(3-4): 172-198.

6. Rabinovich S (2017) Guide to the expression of uncertainty in measurement. $3^{\text {rd }}$ (Edn.), Springer International Publishing, USA.

7. Sedov LI (1993) Similarity and Dimensional Methods in Mechanics. CRC Press, Florida, pp: 496.

8. Sonin AA (2001) The physical basis of dimensional analysis. $2^{\text {nd }}($ Edn.), Department of Mechanical Engineering, MIT, Cambridge.

9. NIST Special Publication 330. The International System of Units (SI). 2008 ${ }^{\text {th }}$ (Edn.), pp: 97.

10. Yarin LP (2012) The Pi-Theorem. Springer-Verlag. Volume 1, Experimental Fluid Mechanics. Berlin.

11. Brillouin L (1964) Scientific uncertainty and information. Academic Press, New York, pp: 178.
12. Murgham H, Miszka D, Wynn K (2019) Simulation of efficiency improvements to an automatic commercial ice maker. ASHRAE Journal 61(5): 36-54.

13. Shah MM (2017) A correlation for heat transfer during boiling on bundles of horizontal plain and enhanced tubes. International Journal of Refrigeration 78: 47-59.

14. Wang S et al. (2018) Vapor-liquid equilibrium and molecular simulation data for carbon dioxide $\left(\mathrm{CO}_{2}\right)+$ trans-1,3,3,3-tetrafluoroprop-1-ene (R1234ze(E)) mixture at temperatures from 283.32 to $353.02 \mathrm{~K}$ and pressures up to 7.6 MPa. International Journal of Refrigeration 98: 362-371.

15. Caliano M, Bianco N, Graditi G, Mongibello L (2019) Experimental and numerical study on a lab-scale latent heat storage prototype for cooling applications. J Phys Conf Ser 1224: 1-13.

16. Bellan S, Alam TE, Gonzalez-Aguilar J, Romero M, Rahman MM, Goswami DY, Stefanakos EK (2015) Numerical and experimental studies on heat transfer characteristics of thermal energy storage system packed with molten salt PCM capsules. Applied Thermal Engineering 90: 970-979.

17. Oreskes N, Shrader-Frechette K, Belitz R (1994) Verification, validation and confirmation of numerical models in the Earth sciences. Science 263(5147): 641-646.

18. Menin B (2015) Possible limits of accuracy in measurement of fundamental physical constants. International Referred Journal of Engineering and Science 4(8): 8-14.

19. Volkenstein MV (2009) Entropy and Information (Birkhauser Verlag AG, Basel-Boston-Berlin).

20. Wasserstein RL, Allen L, Lazar A (2019) Moving to a World Beyond $\mathrm{p}<0.05$. The American Statistician 73(1): 1-19.

21. Wasserstein RL, Schirm AL, Lazar NA (2019) Moving to a World Beyond " $\mathrm{p}<0.05$ ". The American Statistician 73(1): 1-19. 